\title{
Just in Time: Personal Temporal Insights for Altering Model Decisions
}

\author{
Naama Boer \\ Tel Aviv University \\ naamaboer@mail.tau.ac.il
}

\author{
Daniel Deutch \\ Tel Aviv University \\ danielde@post.tau.ac.il
}

\author{
Nave Frost \\ Tel Aviv University \\ navefrost@mail.tau.ac.il
}

\author{
Tova Milo \\ Tel Aviv University \\ milo@post.tau.ac.il
}

\begin{abstract}
The interpretability of complex Machine Learning models is coming to be a critical social concern, as they are increasingly used in human-related decision-making processes such as resume filtering or loan applications. Individuals receiving an undesired classification are likely to call for an explanation - preferably one that specifies what they should do in order to alter that decision when they reapply in the future. Existing work focuses on a single ML model and a single point in time, whereas in practice, both models and data evolve over time: an explanation for an application rejection in 2018 may be irrelevant in 2019 since in the meantime both the model and the applicant's data can change.

To this end, we propose a novel framework that provides users with insights and plans for changing their classification in particular future time points. The solution is based on combining state-of-the-art algorithms for (single) model explanations, ones for predicting future models, and databasestyle querying of the obtained explanations. We propose to demonstrate the usefulness of our solution in the context of loan applications, and interactively engage the audience in computing and viewing suggestions tailored for applicants based on their unique characteristic.
\end{abstract}

\section{INTRODUCTION}

In recent years critical decision-making processes, such as credit score assignment, resume filtering and loan approvals are vastly supported by complex machine learning classifiers. A significant drawback to the utilization of such complex models is their opaqueness, which leaves two important questions unanswered: why did we get a classification result, such as a loan request rejection? What can we do to change it, e.g., what could an applicant do so that his next loan request is approved?

To address this problem, much research has been devoted recently to the explainability of complex Machine Learning models. For example, some solutions [1], [2] identify the most influential features in the input, and others [3] produce approximated interpretable models. These approaches typically provide answers only as to why a decision was made. However, they fall short of providing a practical plan of action for changing the decision. This is in part because they do not account for the temporal aspect of such a plan, namely the evolution of both the user characteristics and the classification models.

Example I.1. To illustrate, consider a bank that uses a Machine Learning model to classify loan application based the following features: Age, Household status, Annual Income, Monthly Debt, Job Seniority and the requested Loan Amount. Further consider an applicant, John, 29 years old, whose loan request is rejected.

John wants to come up with a plan for altering this rejection. By employing a tool such as [1], he is advised to increase his income by 20\%. John works hard to get promoted at work and achieves the desired income within two years. He then reapplies, only to discover that the loan criteria have changed, and his request is again rejected. This time the explanation is that his debt is too high. He wasn't aware that for people over 30, income requirements are often relaxed while debt requirements tend to become stricter. In hindsight, John would rather focus on decreasing his debt over working towards getting a raise.

The suggestions given to the user in this simple example suffer from several deficiencies. They ignore the expected changes in the user's profile (e.g. age increases over time, and often so does seniority), as well as potential changes in the classifier, due to e.g varying data distributions, some of which may be predicted based on past behavior. Furthermore, users differ in their preferences and limitations. For example, one may not be able to increase salary beyond a certain bound, or would rather decrease debt instead. In a dynamic user-focused environment, a satisfactory solution must guide the user in three fundamental aspects: Which features to modify, how to modify them, and when to (re)apply. Following are examples of questions related to these aspects, which are likely to be raised by applicants:

1) No modification: What is the closest time point (if any) at which reapplying without modifications will be APPROVED?

2) Minimal features set: What is the smallest set of features whose modification can lead to APPROVAL? (when? and how should they be modified?).

3) Dominant feature: Is there a single feature whose modification leads to APPROVAL in all future time points? (and how should it be modified at each point?)

4) Minimal overall modification: What is the minimal overall modification (by some distance measure) that leads to APPROVAL, and when?

5) Maximal confidence: Which modifications (and at which time point) would maximize chances of AP- 
PROVAL?

6) Turning point: Is there a time point after which, with some modifications, the confidence of being APPROVED always exceeds $\alpha$ ?

We present JustInTime, a system enabling users to obtain answers to such questions, thereby assisting them in devising a practical plan of action for altering undesired decisions. The system relies on past labeled data with timestamps. An initial configuration is performed by a system administrator through a dedicated admin UI. The administrator sets parameters controlling the amount and time intervals between future time points. According to these parameters the Models Generator processes the training data and trains a sequence of models, each designated for a specific future time span. In addition, the administrator may define global Domain constraints derived from the domain characteristics (such as database integrity constraints), that will be imposed on all users.

User interactions use a dedicated UI and begin with defining preferences and limitations in the form of constraints. The Candidates Generator components then generate a set of modifications candidates for each time point, and store them in the system's database. Each of these candidates represents a potential applicable modification to the user's data profile, that is expected to alter the corresponding model's decision if applied. Users can then query the database through a friendly dedicated interface, consisting of canned questions of the type exemplified earlier (translated to SQL queries) ${ }^{1}$, with the output presented to the user in the form of verbal or graphic insights.

We will demonstrate the operation of Just InTime through a loan application scenario, using the Lending Club Loan Data public dataset containing the details of approximately $1 \mathrm{M}$ loan applications from the years 2007-2018 [4]. Specifically we will demonstrate how applicants whose applications got rejected can obtain actionable insights using a variety of queries. The demonstration will interactively engage the audience who will play the role of rejected applicants, showcasing the system's operation and its different facets.

\section{TECHNICAL OVERVIEW}

In this section, we formally define our framework components and demonstrate their operation. We start by providing the necessary preliminaries for the case of a single decision-making model at the present time. Afterwards, we introduce our temporal framework that consists of two phases. In the first phase, a database of candidate proposals is generated, and in the second phase the user interacts with the data gathered in order to obtain insights for achieving the desired classification.

\section{A. Preliminaries}

We start by recalling some basic notions required for the generation of proposals. A key notion that we use is that of a

\footnotetext{
${ }^{1}$ Experts users may compose additional SQL queries
}

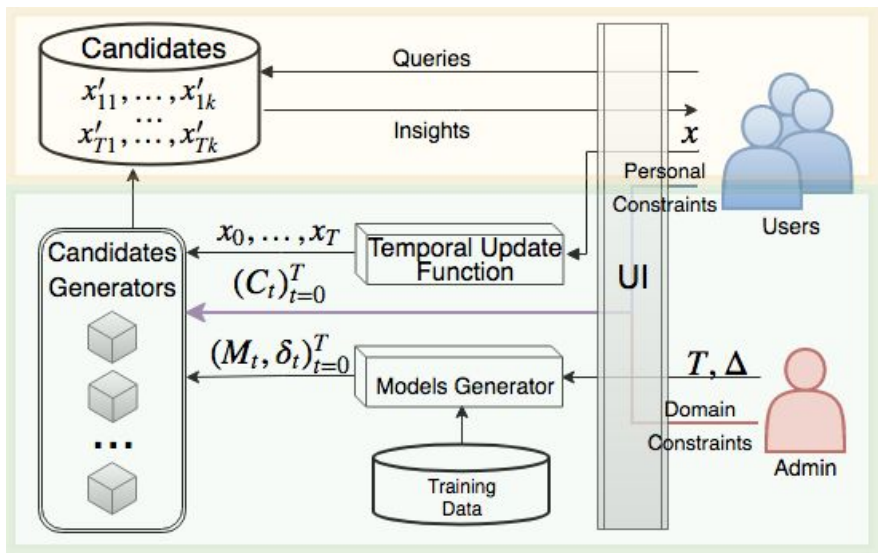

Fig. 1: System Architecture

Machine Learning model. For simplicity, we focus on binary classification, but the framework can be easily generalized to multi-class problems.

Definition II.1. Let $d$ be the dimension of the input space, a Machine Learning model $M: \mathbb{R}^{d} \mapsto[0,1]$ is a function such that $\forall x \in \mathbb{R}^{d}, M(x)$ is the probability of the desired positive classification of the vector $x$.

In our setting, the vector $x$ represents the user's attributes (i.e. profile). For altering a decision, some of these attributes should be modified. However, as observed in [5], in practical settings not all modifications are viable. For example, a person's age can not be decreased, a user may prefer not to change her address, etc. Thus, users may further define a constraints function according to their preferences and limitations.

Definition II.2. A Constraints Function $C$ maps vectors in $\mathbb{R}^{d}$ to a subset of $\mathbb{R}^{d}$. For a given input $x \in \mathbb{R}^{d}$, the set $C(x) \subseteq$ $\mathbb{R}^{d}$ denotes the valid modifications to $x$.

In JustInTime, constraints specified by the administrator and the user are joined to form a general constraints function as defined above. Constraints may refer to a single point in time or all of them, and may contain any number of linear inequalities joined by conjunctions and disjunctions, over any subset of attributes of the input vector. In addition to user attributes, constraints can refer to three special properties: distance from the input w.r.t. $l_{2}$-norm (termed 'diff'), distance w.r.t. $l_{0}$-norm ('gap'), and the overall model score ('confidence') of the given input.

Definition II.3. Given a model $M$, input $x$ and constraints $C$ the set of Decision Altering Candidates is defined as:

$$
A=\left\{x^{\prime} \in \mathbb{R}^{d} \mid x^{\prime} \in C(x) \text { and } M\left(x^{\prime}\right)>\delta\right\} .
$$

Where $\delta$ is the model threshold, i.e. candidates with model score greater then $\delta$ are classified positively.

We base our solution on an algorithm developed in [5] by a subset of the present authors, for finding a candidate $x^{\prime} \in A$ that aims to minimize the distance from the original 
input point $x$. Obtaining an optimal candidate was shown to be an NP-hard problem for e.g Random Forests and Neural Networks, hence the iterative algorithm developed in [5] applies model-dependent heuristics. While convergence is not guaranteed in general, it was shown empirically that the algorithm converges after a small number of iterations. We adjusted the algorithm to our problem by incorporating diverse objectives (confidence, gap and diff) when searching for the candidates, as opposed to a single distance measure. In addition, we output top- $k$ candidates in each iteration, as opposed to just one, using a beam search with width $k$ to prune the least promising candidates.

For reasons explained in the introduction, considering only the modification that would alter the decision in the present model is insufficient, thus in the following subsection we suggest a generalized framework that takes the temporal aspect into consideration.

\begin{tabular}{|c|c|}
\hline $\begin{array}{c}\stackrel{(1)}{ } \text { No } \\
\text { modification }\end{array}$ & $\begin{array}{l}\text { SELECT Min(time) } \\
\text { FROM candidates } \\
\text { WHERE diff }=0\end{array}$ \\
\hline $\begin{array}{l}{ }^{(2)} \text { Minimal } \\
\text { features set }\end{array}$ & $\begin{array}{l}\text { SELECT * } \\
\text { FROM candidates } \\
\text { ORDER BY gap } \\
\text { LIMIT } 1\end{array}$ \\
\hline $\begin{array}{l}{ }^{(3)} \text { Dominant } \\
\text { feature } \\
\text { (income) }\end{array}$ & $\begin{array}{l}\text { SELECT distinct time as } t \\
\text { FROM candidates } \\
\text { WHERE EXISTS } \\
\text { (SELECT * } \\
\text { FROM candidates as cnd } \\
\text { INNER JOIN temporal_inputs as ti } \\
\text { ON ti.time = cnd.time } \\
\text { WHERE cnd.time }=\mathrm{t} \\
\text { AND ((gap = o) OR (gap = 1 } \\
\text { AND cnd.income } !=\text { ti.income))) }\end{array}$ \\
\hline $\begin{array}{c}{ }^{(4)} \text { Minimal } \\
\text { overall } \\
\text { modifications }\end{array}$ & $\begin{array}{l}\text { SELECT Min(diff) } \\
\text { FROM candidates }\end{array}$ \\
\hline $\begin{array}{l}\text { (5) Maximal } \\
\text { confidence }\end{array}$ & $\begin{array}{l}\text { SELECT * } \\
\text { FROM candidates } \\
\text { ORDER BY P DESC } \\
\text { LIMIT } 1\end{array}$ \\
\hline $\begin{array}{l}{ }^{(6)} \text { Turning } \\
\text { time point }\end{array}$ & $\begin{array}{l}\text { SELECT Min(time) } \\
\text { FROM candidates } \\
\text { WHERE time }>=\text { ALL } \\
\text { (SELECT time as } t \\
\text { FROM candidates } \\
\text { WHERE EXISTS }(\ldots) \text { ) }\end{array}$ \\
\hline
\end{tabular}

Fig. 2: Sample of predefined queries

\section{B. Temporal Candidates Generation}

The temporal candidates generation phase is shown in the bottom section of Figure 1. In order to provide users with helpful insights regarding the future, the system has to predict how future models are likely to operate. Generating future models requires labeled training data with timestamps. Additionally, two parameters, $T$ and $\Delta$, determine the time span handled by the system. $T$ is the number of time points considered and $\Delta$ sets the length of the interval between consecutive points. The models generator then uses existing domain adaptation methods [6], in order to create a sequence of pairs $\left(M_{t}, \delta_{t}\right)_{t=0}^{T}$, where $M_{t}$ is the expected approximated model at future time $t$, and $\delta_{t}$ is its threshold. The models generator uses the training data to learn the time variations of the data distribution, using a method from [6], that relies on two techniques: probability distribution embedding into a reproducing kernel Hilbert space, and vector-valued regression. Future models are then trained based on the approximated future distributions. Note that this part of the candidates generation process is performed once and is independent of any specific user.

Recall that some of the attributes (features) are known to change over time (e.g. age increases). Thus, every future model operates on future representations of the features vector, which is generated in our setting using a Temporal Update Function.

Definition II.4. A Temporal Update Function receives a vector $x \in \mathbb{R}^{d}$. For features specified as "non temporal" $f$ is the identity function. For every "temporal" feature $v$, the value of $v$ at time point $t$ is given by $f(x, t)[v]$.

Example II.5. To continue with our running example, age is a temporal feature hence $f(x, 3)[$ age $]=x[$ age $]+3 \Delta$.

As previously mentioned, each user is represented by a vector $x \in \mathbb{R}^{d}$. The temporal update function operates on $x$ and outputs the future temporal representations of $x$, namely $\{f(x, t)\}_{t=0}^{T}$, denoted $x_{0}, \ldots, x_{T}$, for simplicity. These outputs are stored in a relational table called temporal inputs. The input to the candidates generators is then:

- Temporal input vectors $x_{0}, \ldots, x_{T}$ in $\mathbb{R}^{d}$.

- Sequence of model and threshold pairs $\left(M_{t}, \delta_{t}\right)_{t=0}^{T}$.

- Constraints functions $\left(C_{t}\right)_{t=o}^{T}$ which are the conjunction of user and domain constraints.

The core of the temporal candidates generation phase is a sequence of candidates generator components presented in the previous subsection. Each generator is responsible for outputting $k$ decision-altering candidates fitted for the corresponding time point $t \in\{0, \ldots, T\}$, that we denote $x_{t 1}^{\prime}, \ldots, x_{t k}^{\prime}$. The generators are independent of each other, and thus they can be executed in parallel. The results (set of candidates per time point) are then stored in a candidates table, ready to be queried by the user.

Since $A_{t}$, the set of decision altering candidates (Definition II.3) at time $t$, may be arbitrarily large, whereas we are interested in a small, optimized and diverse subset per each time point, we employ the aforementioned adaptation of the algorithm in [5] to select a diverse set of top- $k$ candidates, using several useful predefined metrics. The diversity ensures that limiting the number of candidates does not lead to a degradation in the quality of the answers to user queries.

\section{Obtaining insights for Altering Model Decisions}

The previous phase is concluded with the generation of the candidates table. In this phase the user interacts with the relational database in order to obtain personalized insights. Experts may interact with the system directly in SQL, while for non-experts we provide an expressive and easy-to-use interface for formulating a broad range of 


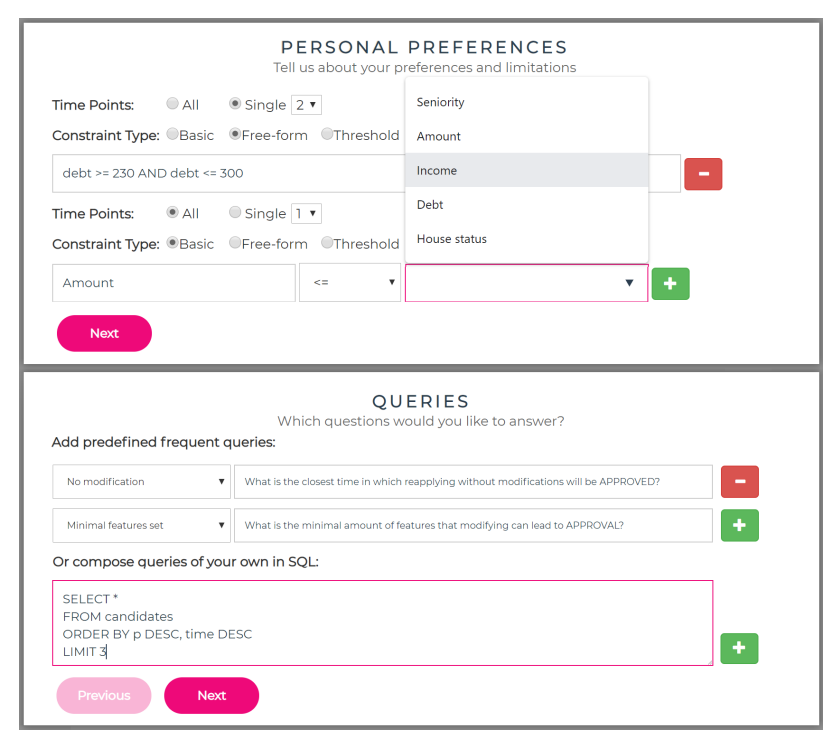

(a) Preferences and Queries Screens

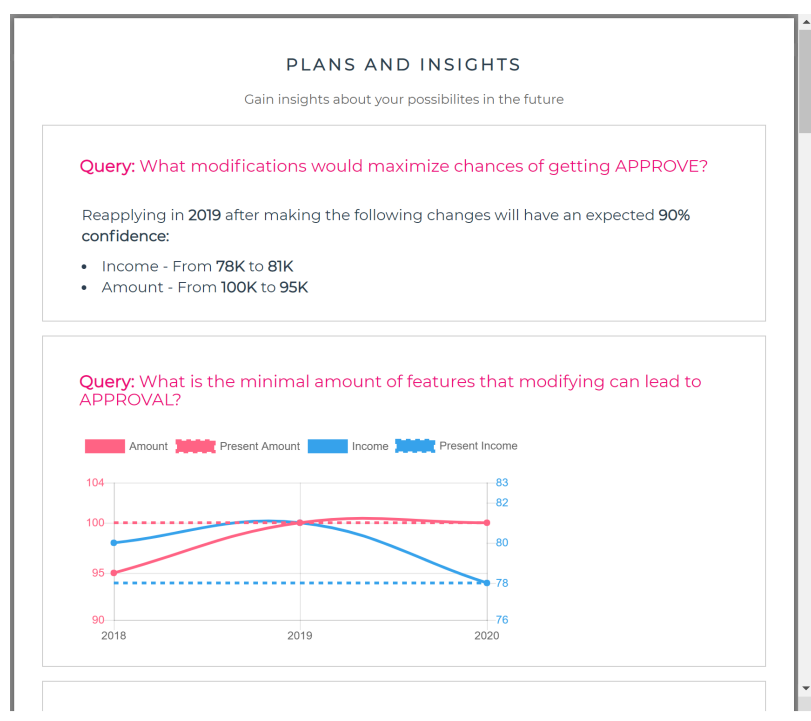

(b) Insights Screen

Fig. 3: User Interface Screen-shots

common questions (including those listed in the introduction), translated by the system into SQL queries. Figure 2 exemplifies several interesting questions along with their corresponding SQL queries.

\section{SYSTEM AND DEMONSTRATION OVERVIEW}

The JustInTime backend is implemented in Python 3.6 and its frontend in JavaScript. It uses MySQL server for storing and querying the generated database. The candidates generator and the models generator components use the $\mathrm{H} 2 \mathrm{O}$ module for data modeling. The models generator trains a random forest classifier for each time span.

The operation of the system is demonstrated w.r.t. the Lending Club Loan Data containing details of approximately $1 M$ loan applications from 2007 to 2018, published in Kaggle [4]. We will demonstrate that Just InTime helps loan applicants to obtain actionable insights on a variety of questions. The demonstration will interactively engage the audience, showcasing the different facets of the system.

We will start by demonstrating the functionality of the system through a reenactment of five real-life loan applications that were denied. The interaction with the system is performed in three steps, each in a designated screen. The audience will suggest preferences and limitations on behalf of the rejected applicants in the Personal Preferences screen. In the Queries screen, we offer the audience a selection of predefined queries, such as the ones listed in the introduction (bottom of Figure 3a). The Plans and Insights screen presents insights with regard to these queries, subject to conditions set in the preferences step (Figure $3 b$ ).

We will continue the demonstration prompting participants to play the role of loan applicants. After setting their features, they will be assigned a score by the present classifier, and further interact with the system in order to attain a plan that achieves their goals. Since the system was designed for non-experts, it requires no prior skills.

To illustrate what happens behind the scenes, we will invite the audience to observe the operation of different stages in our solution. Starting with an excerpt of the raw training data, and continuing with temporal representations of input vectors and generated future models. Lastly, we will examine the execution of a single candidates generator and the candidates it generates.

Related work As the main focus of our work is to provide explanations within a temporal framework, it relates to both Interpretable Machine Learning and Domain Adaptation. A significant part of the work on complex models explainability focuses on explaining particular predictions, often in the context of social accountability and transparency. Within this class of works, the closest to ours are such that use perturbations of the test point and draw conclusions based on how the prediction changes [1], [5], [7]. These solutions mostly promote understanding of a specific decision and engender trust in the model performance, but they are not well suited for attaining an actionable plan for altering the classification, subject to personal and temporal constraints.

Our work also closely relates to the field of Domain Adaptation, specifically the task of predicting future classifiers given past labeled data with timestamps [6], [8]. These are complementary to our work, as we use such solutions as a component of our framework.

\section{ACKNOWLEDGMENT}

This work has been partially funded by the Blavatnik Fund, the Israeli Science Foundation, Intel, and the European Research Council (ERC) under the Europe Unions Horizon 2020 research and innovation programme (grant agreement No. 804302). 


\section{REFERENCES}

[1] A. Datta, S. Sen, and Y. Zick, "Algorithmic transparency via quantitative input influence: Theory and experiments with learning systems," in Symposium on Security and Privacy, 2016.

[2] A. Shrikumar, P. Greenside, and A. Kundaje, "Learning important features through propagating activation differences," arXiv, 2017.

[3] M. T. Ribeiro, S. Singh, and C. Guestrin, "Why should i trust you?: Explaining the predictions of any classifier," in SIGKDD, 2016.

[4] "Lending club loan data," https://tinyurl.com/y7rwt59e, Kaggle.

[5] D. Deutch and N. Frost, "Constraints-based explanations of classifications," in $I C D E, 2019$.

[6] C. H. Lampert, "Predicting the future behavior of a time-varying probability distribution," in CVPR, 2015.

[7] P. Adler, C. Falk, S. A. Friedler, T. Nix, G. Rybeck, C. Scheidegger, B. Smith, and S. Venkatasubramanian, "Auditing black-box models for indirect influence," in Knowledge and Information Systems, 2018.

[8] A. Kumagai and T. Iwata, "Learning future classifiers without additional data." in $A A A I, 2016$. 